\title{
Childhood leg length and adult mortality: follow up of the Carnegie (Boyd Orr) Survey of Diet and Health in Pre-war Britain
}

David J Gunnell, George Davey Smith, Stephen Frankel, Kiran Nanchahal, Fiona E M Braddon, John Pemberton, Tim J Peters

\begin{abstract}
Objective-To investigate the relation between childhood height, its componentsleg length and trunk length-and mortality in adulthood.

Design-Cohort study based on the Carnegie (Boyd Orr) Survey of diet and health in pre-war Britain, 1937-9.

Setting-The 14 centres in England and Scotland that participated in the Carnegie Survey and where children were examined. Scottish centres: Aberdeen, Dundee, West Wemyss, Coaltown of Wemyss, Hopeman, Methlick, Tarves, Barthol Chapel. English Centres: Liverpool, Yorkshire, Barrow in Furness, Wisbech, Fulham, and Bethnal Green.
\end{abstract}

Subjects-2990 boys and girls aged between 2 years and 14 years 9 months when they were examined in 1937-9. These children were drawn from 1134 families who underwent a one week assessment of family diet and home circumstances. Of these, $2547(85 \%)$ have been traced and flagged using the NHS Central Register.

Main outcome measures-Age adjusted overall, coronary heart disease, and cancer mortality in men and women in relation to age and sex specific $z$ scores for height, leg length, and trunk length. All analyses were adjusted for the possible confounding effects of childhood and adult socioeconomic circumstances and childhood diet.

Results-Leg length was the component of childhood height most strongly associated with socioeconomic and dietary exposures. There was no significant relation between childhood height and overall mortality. Height-mortality relations were observed in relation to both coronary heart disease (CHD) and cancer. Leg length was the component of height most strongly related to cause specific mortality. In men and women CHD mortality increased with decreasing childhood leg length. Men in the lowest leg length quintile had a relative risk (RR) of $2.5(95 \%$ CI 1.0 to 6.2 ) compared to those with the longest legs (linear trend $p=0.14$ ). Similarly, women in the lowest leg length quintile had a RR of 3.9 (95\% CI 0.8 to 19.0 ; linear trend $p<0.01)$. Adjustment for childhood and adult socioeconomic circumstances had little effect on these trends. In men, but not women, those who as children had long legs experienced increased cancer mortality. The significant relations between anthropometry and both CHD and cancer mortality were restricted to those aged $<8$ years when measured.

Conclusions-These findings suggest that adverse diet and living conditions in childhood, for which leg length seems to be a particularly sensitive indicator, are associated with increased risk of CHD in adulthood and possibly reduced cancer risk. It is likely that these influences operate after birth, during the first few years of life.

(F Epidemiol Community Health 1998;52:142-152)

For many centuries it has been recognised that a child's environment and diet are important in determining its rate of growth, timing of maturation, and final stature. ${ }^{1}$ In the past 60 years epidemiological analyses have shown that poverty, undernutrition, and disease exposure in childhood may also have important long term effects on adult health and life expectancy. ${ }^{2}$ Adult height reflects a child's nutrition and health throughout its growing years and, although final height is limited by a child's genotype, environmental influences also affect its adult size. ${ }^{3}$ Exposure to the factors that inhibit or promote growth - nutrition, disease, psychological stress, overcrowding, and other socioeconomic factors-differs both within and between populations.

A number of cohort studies in men and women have found striking inverse relations between adult height and cardiovascular disease. $^{4-9}$ The relation between height and cancer is quite different. A number of cohort studies report an increased risk of breast, colorectal, and other cancers in taller people. $^{61011}$

It has been argued that height-mortality relations seen in adults are distorted by the effects that pre-existing illness may have on height. Such views are supported by the reduction in strength of the height-mortality relation over time $^{6}$ and the absence of a relation in studies with long follow up. ${ }^{12}$ One way to further investigate such explanations is to examine the mortality experience of cohorts where height measurements were taken in childhood or early adulthood, rather than in middle age. ${ }^{13-15}$ Results from these studies are not consistent and the only study to examine 


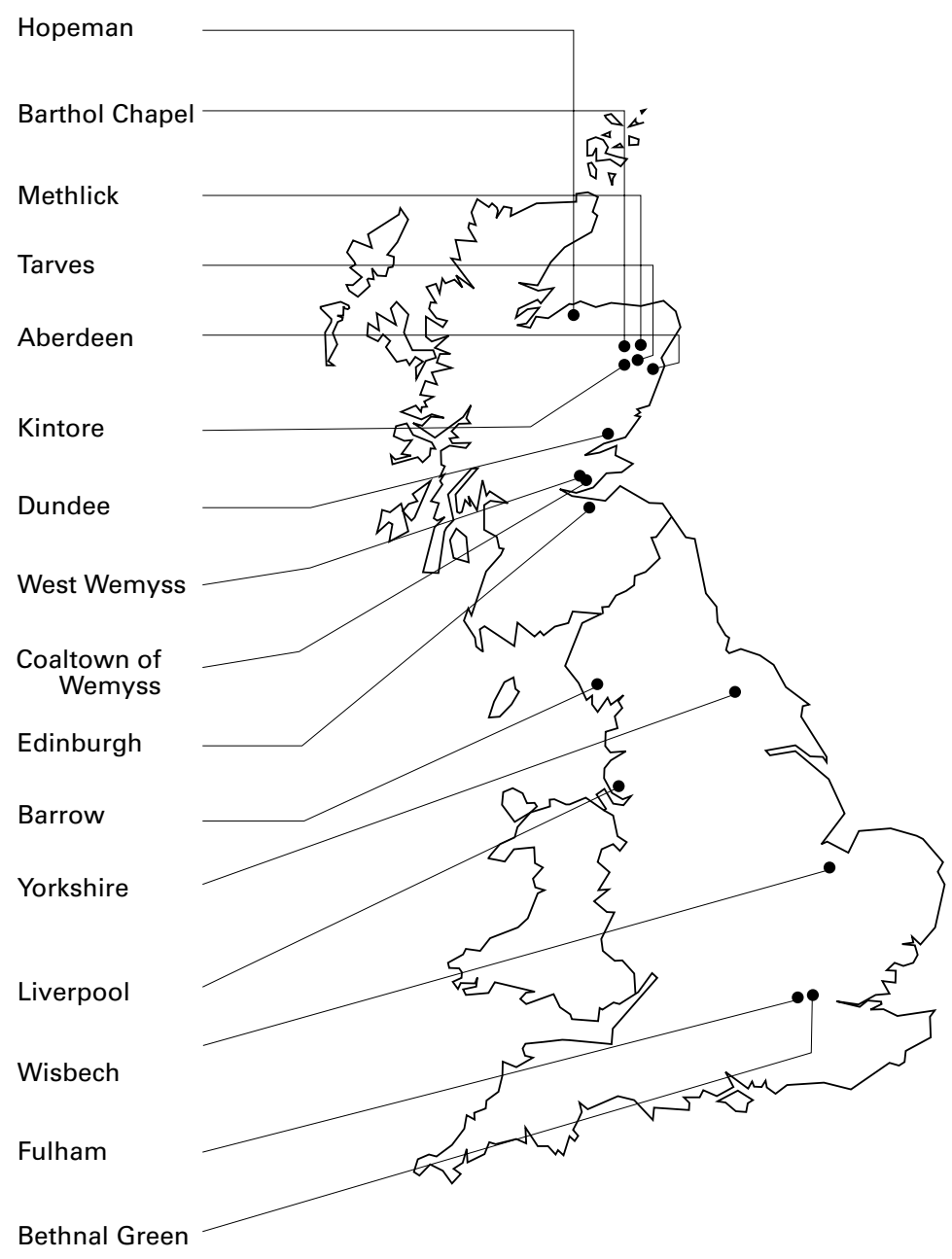

Figure 1 Survey centres used in the Carnegie Survey 1937-9.

height in childhood and adult mortality did not find a significant relation with all cause mortality in either pre- or post-pubertal children. ${ }^{13}$

Over the past 100 years there have been considerable increases in the height of children and adults in the developed world. ${ }^{16}$ Cross sectional studies of Japanese children measured over a 20 year period suggest that the greater part of the secular increase in height in that country is due to an increase in leg length, rather than a symmetric increase in leg and trunk growth. ${ }^{17}$ This is supported by findings from other cross sectional studies that show that social class and geographical differences in height are mainly due to differences in leg length. ${ }^{18}{ }^{19}$ Few prospective studies have examined the influence of the components of height on adult mortality. ${ }^{2021}$ One found that cancer mortality increased with increases in height in men and women - almost all the observed relation being due to differences in leg length rather than sitting height. ${ }^{20}$ In another there was little difference in mean leg length between cancer cases and controls. ${ }^{21}$ We know of no studies that have prospectively examined the relation between leg length and coronary heart disease (CHD) mortality although the relation between body proportions and $\mathrm{CHD}$ has attracted some interest in the past. $^{22}{ }^{23}$
We have examined the relation between the components of height in childhood-leg length and trunk length - and adult mortality in a cohort study, based on the Carnegie Survey of Diet and Health in Pre-War Britain. ${ }^{24}{ }^{25}$ Cross sectional analyses of the Carnegie (Boyd Orr) Survey have shown that leg length was consistently better than overall height for indicating children's socioeconomic group. ${ }^{26}$ In 1951 Isabella Leitch, a nutritionist who was involved in the planning of the survey, commented that " ...there is a physiological basis for preferring tall and long legged people because, in general, that type represents completion of growth and appears to connote a certain superiority of constitution." ${ }^{26}$ This paper tests Isabella Leitch's hypothesis and investigates the relation between childhood height, its components-leg length and trunk lengthand adult mortality.

\section{Methods}

THE CARNEGIE (BOYD ORR) SURVEY

The methods used in the Carnegie Survey, the tracing of the original survey members and the representativeness of those traced and flagged on the National Health Service (NHS) Central Register have already been described..$^{25}$ Briefly, the material used for this analysis is drawn from the original records of the Carnegie Survey of Family Diet and Health in Pre-War Britain. ${ }^{24}$ These records were retrieved from the Rowett Research Institute in 1988 and information contained within these records (name, age, and address in 1937-9) was used to trace the children who took part in the survey. The survey was carried out in 16 centres in England and Scotland over a two year period between 1937 and 1939 (fig 1). These centres were chosen to give a mix of contemporary rural and urban conditions. Families who were willing to participate in a one week survey of household food consumption were generally identified from the more deprived localities in these centres through contacts made by local health workers. Some families from more affluent backgrounds were recruited separately by the survey team. Most of the families who were approached consented to participate and most of them completed the one week dietary diary.

Altogether 1352 families provided detailed information on household socioeconomic circumstances and diet. Occupation of the head of household was recorded, as were details of family composition and total family expenditure on food. In all but two of the survey centres (Edinburgh and Kintore) the children from the families underwent detailed physical examination. This included measures of height, leg length (cristal height), weight, shoulder breadth, haemoglobin, and an assessment of the general health of the child. According to the original survey report, 3762 children were examined. The records for most of these children have been found ${ }^{25}$ and the NHS Central Register has been used to follow them up for mortality. 
SAMPLE USED IN THIS ANALYSIS

Only survey members aged 2 years to 14 years 9 months when examined $(n=2990)$ are included in these analyses. Height measurements in the younger children are less complete and unreliable and over the age of 14 years 9 months the number of children is small $(n=159)$. The school leaving age was not raised to 15 until 1945 and therefore the older children are probably unrepresentative. All of the survey members who have been traced and who were alive and resident in the British Isles on the 1 January 1948 are included in the mortality analyses, which are based on deaths occurring up to 31 July 1995.

\section{ANTHROPOMETRY}

In all but one of the 14 survey centres the measurements were taken by members of a three person medical examination team. In Aberdeen, the pilot centre for the study, examinations were performed by a different team. Standing height was measured to the nearest millimetre with a portable measuring stand. ${ }^{24}$ Leg length was measured with a steel tape measure and recorded to the nearest millimetre as the distance from the ground to the summit of the iliac crest ("cristal height"). ${ }^{24} \mathrm{No}$ shoes or socks were worn for these measurements. Trunk length has been calculated by subtracting leg length from overall height. The socioeconomic background of the children was not specifically drawn to the attention of those who undertook the examinations.

All anthropometric variables have been converted to standard deviation scores ( $\mathrm{z}$ scores). These express a child's measurement as the number of standard deviations its value is from the mean for its age and sex. The $\mathrm{z}$ score thus provides a means of comparison across groups which is independent of age and sex.

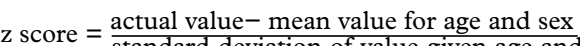
standard deviation of value given age and sex

Within each six month age and sex band an assessment of the goodness of fit to a Gaussian distribution of the anthropometric index (height, leg length, trunk length) was made using the Shapiro-Wilk test for normality. In only $11(7.1 \%)$ of these 156 age/sex bands for height, leg length, and trunk length did the distribution differ significantly $(\mathrm{p}<0.05)$ from normal; this assumption was therefore considered reasonable throughout the analyses. Cubic polynomial regression models were used to estimate expected values for the anthropometric measures in males and females separately for children aged 2 years to 14 years 9 months based on the complete dataset. ${ }^{28}$ The only explanatory variables used in these models were age in months, age squared, and age cubed. Although in some models the addition of a cubic term did not improve the fit of the model, this term was included throughout for consistency. Standard deviations for the anthropometric measures within six month age groups did not follow a smooth pattern across age groups. This was because of the relatively small numbers in each age group. As this could distort $\mathrm{z}$ scores, polynomial regression models were used to smooth the age specific standard deviations. Standard deviation was modelled on age by dividing the cohort into six month age bands and calculating the standard deviations for the anthropometric variable within each of these bands.

Generally it is preferable to use an external standard reference population for the creation of $\mathrm{z}$ scores and assessing the relative nutritional status of populations. In our main analyses we have used internally derived standards for two reasons. Firstly, secular trends in heights and reduction in the age of the adolescent growth spurt mean that if the 1966 British reference standards for children's heights are used these are likely to result in exaggeratedly negative $\mathrm{z}$ scores in the survey children aged around the time of the adolescent growth spurt. ${ }^{29}$ No acceptable cross sectional reference standards exist for children in the 1930s. The second reason was that representative British standards for leg length and trunk length do not exist. However, to ensure that the use of internal standards did not bias our final results, $\mathrm{z}$ scores for the height of survey members were also calculated using the British 1966 growth standards $^{29}$ and $\mathrm{z}$ scores for leg length (cristal height) from American reference data. ${ }^{30}$

Table 1 Pearson's correlation coefficients between anthropometry, childhood dietary, and socioeconomic variables and adult socioeconomic status

\begin{tabular}{|c|c|c|c|}
\hline Anthropometric, dietary or socioeconomic index (n) & $z$ score for height & $\begin{array}{l}z \text { score for leg } \\
\text { lengthy }\end{array}$ & $\begin{array}{l}z \text { score for trunk } \\
\text { lengthS }\end{array}$ \\
\hline \multicolumn{4}{|l|}{ Men } \\
\hline Birth order (1397) & $-0.14^{\star}$ & $-0.14^{\star}$ & $-0.06^{\star}$ \\
\hline No of children (1394) & $-0.25^{\star}$ & $-0.24^{\star}$ & $-0.15^{\star}$ \\
\hline Per capita food expenditure (1394) & $0.31^{\star}$ & $0.31^{\star}$ & $0.17^{\star}$ \\
\hline Weighted per capita food expenditure (1394) & $0.28^{\star}$ & $0.28^{\star}$ & $0.15^{\star}$ \\
\hline Social class of head of household $+\neq(1287)$ & $-0.18^{\star}$ & $-0.21^{\star}$ & -0.05 \\
\hline Relative family per capita calorie consumption (1394) & $0.23^{\star}$ & $0.26^{\star}$ & $0.08^{\star}$ \\
\hline Townsend score (1182) & $-0.06^{\star}$ & $-0.09^{\star}$ & -0.01 \\
\hline \multicolumn{4}{|l|}{ Women } \\
\hline Birth order (1556) & $-0.14^{\star}$ & $-0.14^{\star}$ & $-0.06^{\star}$ \\
\hline No of children (1551) & $-0.21^{\star}$ & $-0.21^{\star}$ & $-0.10^{\star}$ \\
\hline Per capita food expenditure (1551) & $0.26^{\star}$ & $0.25^{\star}$ & $0.15^{\star}$ \\
\hline Weighted per capita food expenditure (1551) & $0.25^{\star}$ & $0.23^{\star}$ & $0.15^{\star}$ \\
\hline Social class of head of household $† \neq(1403)$ & $-0.17^{\star}$ & $-0.17^{\star}$ & $-0.08^{\star}$ \\
\hline Relative family per capita calorie consumption (1551) & $0.20^{\star}$ & $0.23^{\star}$ & $0.05^{\star}$ \\
\hline Townsend score $(1296)$ & $-0.04^{\star}$ & -0.04 & -0.01 \\
\hline
\end{tabular}

+Spearman's rank correlation coefficient used, excludes cohort members with unclassifiable occupations. $¥$ Social class categorised as I/ II;III;IV;V; unemployed. \Among the boys 44 fewer individuals had leg length recorded and 46 fewer had values for trunk than those for overall height. For girls 47 fewer had leg length recorded and 53 fewer trunk length. ${ }^{\star} \mathrm{p}<0.05$. 
Table 2 Age adjusted death rates per 1000 person years, with their standard errors (SE) and number of deaths, by quintile of height, leg length and trunk length for men and women

\begin{tabular}{|c|c|c|c|c|c|c|c|c|c|c|}
\hline & & Overa & eight & & Leg le & & & Trunk & gth & \\
\hline $\operatorname{Men}(n=1129)$ & $\begin{array}{l}\text { Quintile of height, leg } \\
\text { length or trunk } z \text { scores }\end{array}$ & Rate & $S E$ & $\begin{array}{l}\text { No of } \\
\text { deaths }\end{array}$ & Rate & $S E$ & $\begin{array}{l}\text { No of } \\
\text { deaths }\end{array}$ & Rate & $S E$ & $\begin{array}{l}\text { No of } \\
\text { deaths }\end{array}$ \\
\hline All causes & 1 (short) & 4.6 & $(0.7)$ & 43 & 4.5 & $(0.7)$ & 45 & 3.9 & $(0.7)$ & 38 \\
\hline & 2 & 4.5 & $(0.7)$ & 45 & 4.3 & $(0.7)$ & 43 & 4.1 & $(0.6)$ & 42 \\
\hline & 3 & 3.6 & $(0.6)$ & 34 & 3.9 & $(0.7)$ & 35 & 4.2 & $(0.7)$ & 40 \\
\hline & 4 & 3.7 & $(0.6)$ & 35 & 4.5 & $(0.7)$ & 45 & 3.7 & $(0.6)$ & 38 \\
\hline & 5 (tall) & 5.2 & $(0.8)$ & 50 & 4.2 & $(0.7)$ & 39 & 5.2 & $(0.8)$ & 49 \\
\hline & total & 4.2 & $(0.3)$ & 207 & 4.2 & $(0.3)$ & 207 & 4.2 & $(0.3)$ & 207 \\
\hline Cardiovascular disease & 1 (short) & 2.9 & $(0.6)$ & 25 & 2.7 & $(0.5)$ & 28 & 2.4 & $(0.5)$ & 23 \\
\hline & 2 & 2.2 & $(0.5)$ & 22 & 2.3 & $(0.5)$ & 23 & 2.0 & $(0.4)$ & 20 \\
\hline & 3 & 2.6 & $(0.6)$ & 24 & 2.0 & $(0.5)$ & 19 & 2.2 & $(0.5)$ & 21 \\
\hline & 4 & 1.5 & $(0.4)$ & 14 & 2.4 & $(0.5)$ & 23 & 1.9 & $(0.4)$ & 19 \\
\hline & 5 (tall) & 2.2 & $(0.5)$ & 21 & 1.4 & $(0.4)$ & 13 & 2.3 & $(0.5)$ & 23 \\
\hline & total & 2.1 & $(0.2)$ & 106 & 2.1 & $(0.2)$ & 106 & 2.1 & $(0.2)$ & 106 \\
\hline Coronary heart disease & 1 (short) & 2.0 & $(0.5)$ & 17 & 1.8 & $(0.4)$ & 18 & 1.6 & $(0.4)$ & 16 \\
\hline & 2 & 1.6 & $(0.4)$ & 16 & 1.6 & $(0.4)$ & 16 & 1.3 & $(0.4)$ & 13 \\
\hline & 3 & 2.0 & $(0.5)$ & 17 & 1.5 & $(0.4)$ & 15 & 1.9 & $(0.5)$ & 18 \\
\hline & 4 & 1.3 & $(0.4)$ & 12 & 2.0 & $(0.5)$ & 19 & 1.5 & $(0.4)$ & 15 \\
\hline & 5 (tall) & 1.5 & $(0.4)$ & 15 & 0.9 & $(0.3)$ & 9 & 1.5 & $(0.4)$ & 15 \\
\hline & total & 1.6 & $(0.2)$ & 77 & 1.6 & $(0.2)$ & 77 & 1.6 & $(0.2)$ & 77 \\
\hline Non-cardiovascular, smoking related mortality & 1 (short) & 0.2 & $(0.1)$ & 3 & 0.4 & $(0.2)$ & 4 & 0.4 & $(0.2)$ & 4 \\
\hline & 2 & 1.0 & $(0.3)$ & 10 & 0.8 & $(0.3)$ & 7 & 0.7 & $(0.3)$ & 7 \\
\hline & 3 & 0.6 & $(0.2)$ & 6 & 1.1 & $(0.4)$ & 9 & 0.5 & $(0.3)$ & 4 \\
\hline & 4 & 0.6 & $(0.2)$ & 6 & 0.8 & $(0.3)$ & 8 & 1.0 & $(0.3)$ & 10 \\
\hline & 5 (tall) & 1.3 & $(0.4)$ & 13 & 1.0 & $(0.3)$ & 10 & 1.4 & $(0.4)$ & 13 \\
\hline & total & 0.8 & $(0.1)$ & 38 & 0.8 & $(0.1)$ & 38 & 0.8 & $(0.1)$ & 38 \\
\hline All cancers & 1 (short) & 0.7 & $(0.3)$ & 7 & 0.9 & $(0.3)$ & 9 & 0.6 & $(0.3)$ & 6 \\
\hline & 2 & 1.3 & $(0.3)$ & 13 & 0.8 & $(0.3)$ & 7 & 1.3 & $(0.4)$ & 14 \\
\hline & 3 & 0.7 & $(0.3)$ & 7 & 1.0 & $(0.3)$ & 9 & 0.7 & $(0.3)$ & 7 \\
\hline & 4 & 0.8 & $(0.3)$ & 8 & 1.1 & $(0.3)$ & 12 & 1.0 & $(0.3)$ & 10 \\
\hline & 5 (tall) & 1.6 & $(0.4)$ & 16 & 1.5 & $(0.4)$ & 14 & 1.5 & $(0.4)$ & 14 \\
\hline & total & 1.1 & $(0.2)$ & 51 & 1.1 & $(0.2)$ & 51 & 1.1 & $(0.2)$ & 51 \\
\hline Non-smoking related cancers & 1 (short) & 0.5 & $(0.2)$ & 5 & 0.5 & $(0.2)$ & 5 & 0.3 & $(0.2)$ & 3 \\
\hline & 2 & 0.3 & $(0.2)$ & 3 & 0.2 & $(0.1)$ & 2 & 0.6 & $(0.3)$ & 7 \\
\hline & 3 & 0.3 & $(0.2)$ & 3 & 0.3 & $(0.2)$ & 3 & 0.3 & $(0.1)$ & 4 \\
\hline & 4 & 0.5 & $(0.2)$ & 5 & 0.6 & $(0.2)$ & 6 & 0.3 & $(0.2)$ & 3 \\
\hline & 5 (tall) & 0.4 & $(0.2)$ & 4 & 0.5 & $(0.3)$ & 4 & 0.3 & $(0.2)$ & 3 \\
\hline & total & 0.4 & $(0.1)$ & 20 & 0.4 & $(0.1)$ & 20 & 0.4 & $(0.1)$ & 20 \\
\hline Other causes & 1 (short) & 1.1 & $(0.3)$ & 11 & 0.8 & $(0.3)$ & 8 & 0.9 & $(0.3)$ & 9 \\
\hline & 2 & 1.0 & $(0.3)$ & 10 & 1.2 & $(0.4)$ & 13 & 0.8 & $(0.3)$ & 8 \\
\hline & 3 & 0.2 & $(0.1)$ & 3 & 0.9 & $(0.3)$ & 7 & 1.3 & $(0.4)$ & 12 \\
\hline & 4 & 1.2 & $(0.4)$ & 12 & 0.9 & $(0.3)$ & 9 & 0.7 & $(0.3)$ & 8 \\
\hline & 5 (tall) & 1.4 & $(0.4)$ & 13 & 1.3 & $(0.4)$ & 12 & 1.4 & $(0.4)$ & 12 \\
\hline & total & 1.0 & $(0.1)$ & 49 & 1.0 & $(0.1)$ & 49 & 1.0 & $(0.1)$ & 49 \\
\hline Women $(n=1195)$ & & & & & & & & & & \\
\hline All causes & 1 (short) & 3.9 & $(0.6)$ & 42 & 3.2 & $(0.5)$ & 37 & 3.5 & $(0.6)$ & 39 \\
\hline & 2 & 2.6 & $(0.5)$ & 29 & 3.1 & $(0.6)$ & 33 & 2.2 & $(0.5)$ & 24 \\
\hline & 3 & 2.8 & $(0.5)$ & 30 & 3.2 & $(0.6)$ & 33 & 3.5 & $(0.6)$ & 36 \\
\hline & 4 & 2.5 & $(0.5)$ & 27 & 2.2 & $(0.5)$ & 22 & 2.5 & $(0.5)$ & 27 \\
\hline & 5 (tall) & 1.9 & $(0.4)$ & 20 & 2.2 & $(0.5)$ & 23 & 2.1 & $(0.5)$ & 22 \\
\hline & total & 2.7 & $(0.2)$ & 148 & 2.7 & $(0.2)$ & 148 & 2.7 & $(0.2)$ & 148 \\
\hline Cardiovascular disease & 1 (short) & 1.5 & $(0.4)$ & 17 & 1.3 & $(0.3)$ & 15 & 0.9 & $(0.3)$ & 11 \\
\hline & 2 & 1.0 & $(0.3)$ & 11 & 1.2 & $(0.3)$ & 13 & 0.6 & $(0.2)$ & 7 \\
\hline & 3 & 0.8 & $(0.3)$ & 9 & 1.1 & $(0.3)$ & 11 & 1.6 & $(0.4)$ & 15 \\
\hline & 4 & 0.7 & $(0.3)$ & 7 & 0.8 & $(0.3)$ & 7 & 0.7 & $(0.3)$ & 8 \\
\hline & 5 (tall) & 0.6 & $(0.2)$ & 6 & 0.4 & $(0.2)$ & 4 & 0.9 & $(0.3)$ & 9 \\
\hline & total & 0.9 & $(0.1)$ & 50 & 0.9 & $(0.1)$ & 50 & 0.9 & $(0.1)$ & 50 \\
\hline Coronary heart disease & 1 (short) & 1.2 & $(0.4)$ & 12 & 0.9 & $(0.3)$ & 10 & 0.4 & $(0.2)$ & 5 \\
\hline & 2 & 0.4 & $(0.2)$ & 4 & 0.7 & $(0.3)$ & 8 & 0.5 & $(0.2)$ & 5 \\
\hline & 3 & 0.4 & $(0.2)$ & 4 & 0.3 & $(0.2)$ & 4 & 1.0 & $(0.3)$ & 9 \\
\hline & 4 & 0.6 & $(0.3)$ & 5 & 0.2 & $(0.2)$ & 2 & 0.4 & $(0.3)$ & 4 \\
\hline & 5 (tall) & 0.1 & $(0.1)$ & 1 & 0.2 & $(0.2)$ & 2 & 0.3 & $(0.2)$ & 3 \\
\hline & total & 0.5 & $(0.1)$ & 26 & 0.5 & $(0.1)$ & 26 & 0.5 & $(0.1)$ & 26 \\
\hline Non-cardiovascular, smoking related mortality & 1 (short) & 0.8 & $(0.3)$ & 8 & 0.5 & $(0.2)$ & 6 & 0.7 & $(0.3)$ & 8 \\
\hline & 2 & 0.3 & $(0.2)$ & 4 & 0.5 & $(0.2)$ & 6 & 0.5 & $(0.3)$ & 5 \\
\hline & 3 & 0.3 & $(0.2)$ & 3 & 0.7 & $(0.3)$ & 8 & 0.3 & $(0.2)$ & 4 \\
\hline & 4 & 0.7 & $(0.3)$ & 8 & 0.3 & $(0.2)$ & 3 & 0.9 & $(0.3)$ & 9 \\
\hline & 5 (tall) & 0.5 & $(0.2)$ & 5 & 0.4 & $(0.2)$ & 5 & 0.2 & $(0.1)$ & 2 \\
\hline & total & 0.5 & $(0.1)$ & 28 & 0.5 & $(0.1)$ & 28 & 0.5 & $(0.1)$ & 28 \\
\hline All cancers & 1 (short) & 1.4 & $(0.4)$ & 15 & 1.0 & $(0.3)$ & 12 & 1.3 & $(0.4)$ & 15 \\
\hline & 2 & 0.9 & $(0.3)$ & 11 & 1.3 & $(0.3)$ & 14 & 1.0 & $(0.3)$ & 10 \\
\hline & 3 & 1.1 & $(0.3)$ & 12 & 1.1 & $(0.3)$ & 12 & 1.2 & $(0.3)$ & 14 \\
\hline & 4 & 1.1 & $(0.3)$ & 12 & 0.9 & $(0.3)$ & 10 & 1.2 & $(0.3)$ & 13 \\
\hline & 5 (tall) & 1.0 & $(0.3)$ & 10 & 1.1 & $(0.3)$ & 12 & 0.8 & $(0.3)$ & 8 \\
\hline & total & 1.1 & $(0.1)$ & 60 & 1.1 & $(0.1)$ & 60 & 1.1 & $(0.1)$ & 60 \\
\hline Non-smoking related cancers & 1 (short) & 0.8 & $(0.3)$ & 9 & 0.7 & $(0.3)$ & 8 & 0.8 & $(0.3)$ & 9 \\
\hline & 2 & 0.7 & $(0.2)$ & 7 & 0.8 & $(0.3)$ & 8 & 0.6 & $(0.2)$ & 6 \\
\hline & 3 & 0.9 & $(0.3)$ & 10 & 0.7 & $(0.3)$ & 7 & 0.9 & $(0.3)$ & 11 \\
\hline & 4 & 0.6 & $(0.3)$ & 6 & 0.6 & $(0.2)$ & 7 & 0.6 & $(0.2)$ & 7 \\
\hline & 5 (tall) & 0.7 & $(0.3)$ & 7 & 0.9 & $(0.3)$ & 9 & 0.6 & $(0.3)$ & 6 \\
\hline & total & 0.7 & $(0.1)$ & 39 & 0.7 & $(0.1)$ & 39 & 0.7 & $(0.1)$ & 39 \\
\hline Other causes & 1 (short) & 0.9 & $(0.3)$ & 10 & 0.9 & $(0.3)$ & 10 & 1.3 & $(0.4)$ & 13 \\
\hline & 2 & 0.7 & $(0.3)$ & 7 & 0.7 & $(0.3)$ & 6 & 0.6 & $(0.3)$ & 7 \\
\hline & 3 & 0.8 & $(0.3)$ & 9 & 1.0 & $(0.3)$ & 10 & 0.7 & $(0.3)$ & 7 \\
\hline & 4 & 0.7 & $(0.3)$ & 8 & 0.6 & $(0.3)$ & 5 & 0.6 & $(0.3)$ & 6 \\
\hline & 5 (tall) & 0.3 & $(0.2)$ & 4 & 0.6 & $(0.2)$ & 7 & 0.5 & $(0.2)$ & 5 \\
\hline & total & 0.7 & $(0.1)$ & 38 & 0.7 & $(0.1)$ & 38 & 0.7 & $(0.1)$ & 38 \\
\hline
\end{tabular}


Table 3 Overall and cause specific mortality hazard ratios (95\% CI) for men and women in relation to a one standard deviation increase in overall height, leg length, and trunk length

\begin{tabular}{|c|c|c|c|c|}
\hline Cause of death & $\begin{array}{l}\text { Number of } \\
\text { deaths }\end{array}$ & Height & Leg length & Trunk length \\
\hline \multicolumn{5}{|l|}{ Men } \\
\hline All causes & 207 & $1.03(0.89,1.19)$ & $0.93(0.79,1.10)$ & $1.15(0.98,1.35)$ \\
\hline Cardiovascular† & 106 & $0.88(0.72,1.07)$ & $0.80(0.64,1.01)$ & $1.11(0.89,1.39)$ \\
\hline Coronary heart disease $\ddagger$ & 77 & $0.88(0.69,1.11)$ & $0.82(0.63,1.07)$ & $1.09(0.84,1.41)$ \\
\hline Non-cardiovascular, smoking related $\rrbracket$ & 38 & $1.36(0.99,1.88)$ & $1.13(0.77,1.67)$ & $1.28(0.88,1.88)$ \\
\hline Cancerף & 51 & $1.32(1.00,1.74)^{\star}$ & $1.23(0.88,1.71)$ & $1.12(0.80,1.57)$ \\
\hline Non-smoking related cancer ${ }^{\star \star \star}$ & 20 & $1.21(0.78,1.88)$ & $1.26(0.75,2.12)$ & $0.96(0.56,1.65)$ \\
\hline Other causes & 49 & $1.08(0.80,1.44)$ & $0.94(0.67,1.30)$ & $1.23(0.89,1.70)$ \\
\hline \multicolumn{5}{|l|}{ Women } \\
\hline All causes & 148 & $0.89(0.74,1.06)$ & $0.94(0.78,1.13)$ & $0.91(0.75,1.09)$ \\
\hline Cardiovasculart & 50 & $0.74(0.55,1.00)$ & $0.68(0.50,0.94)^{\star}$ & $1.06(0.77,1.45)$ \\
\hline Coronary heart disease $\ddagger$ & 26 & $0.56(0.37,0.86)^{\star \star}$ & $0.53(0.34,0.83)^{\star \star}$ & $1.01(0.65,1.57)$ \\
\hline Non-cardiovascular, smoking related $\$ & 28 & $1.01(0.67,1.53)$ & $1.08(0.69,1.66)$ & $0.89(0.58,1.38)$ \\
\hline Cancer $\sqrt{-}$ & 60 & $1.01(0.76,1.34)$ & $1.07(0.80,1.44)$ & $0.95(0.71,1.27)$ \\
\hline Non-smoking related cancer ${ }^{\star \star \star}$ & 39 & $1.04(0.74,1.47)$ & $1.11(0.77,1.59)$ & $0.98(0.68,1.39)$ \\
\hline Other causes & 38 & $0.92(0.65,1.30)$ & $1.14(0.79,1.65)$ & $0.71(0.49,1.04)$ \\
\hline
\end{tabular}

IICD: $390-459$

¥ICD: $410-414$.

§ICD: $140,141,143-9,150,157,160-3,188-9,480-486,491,492,496,531-534$

IICD: $140-209$

${ }^{\star \star \star}$ ICD: $140-209$, except: $140,141,143-9,150,157,160-3,188-9$.

${ }^{\star} \mathrm{p}<0.05 ;{ }^{\star \star} \mathrm{p}<0.01$.

Table 4 Overall and cause specific mortality hazard ratios (95\% CI) for men and women in relation to a one standard deviation increase in overall height, leg length, and trunk length adjusted for indices of childhood socioeconomic deprivation, calorie consumption, and birth order, and adult Townsend score

\begin{tabular}{lllll}
\hline Cause of death & $\begin{array}{l}\text { No of } \\
\text { deaths }\end{array}$ & Height & Leg length & Trunk length \\
\hline Men $(n=1129)$ & & & & \\
All causes & 207 & $1.06(0.92,1.24)$ & $0.97(0.82,1.15)$ & $1.14(0.97,1.34)$ \\
Cardiovascular & 106 & $0.85(0.69,1.06)$ & $0.79(0.62,1.00)^{\star}$ & $1.09(0.87,1.37)$ \\
Coronary heart disease & 77 & $0.84(0.65,1.08)$ & $0.79(0.60,1.04)$ & $1.07(0.82,1.39)$ \\
Non-cardiovascular, smoking related & 38 & $1.49(1.06,2.11)^{\star}$ & $1.33(0.88,2.01)$ & $1.20(0.80,1.79)$ \\
Cancer & 51 & $1.42(1.05,1.91)^{\star}$ & $1.38(0.98,1.96)$ & $1.07(0.76,1.50)$ \\
Non-smoking related cancer & 20 & $1.30(0.80,2.11)$ & $1.44(0.83,2.49)$ & $0.90(0.51,1.57)$ \\
Other causes & 49 & $1.20(0.87,1.64)$ & $1.03(0.73,1.47)$ & $1.26(0.91,1.75)$ \\
Women $(n=1195)$ & & & & \\
All causes & 148 & $0.92(0.77,1.11)$ & $0.97(0.80,1.17)$ & $0.93(0.77,1.12)$ \\
Cardiovascular & 50 & $0.81(0.59,1.10)$ & $0.72(0.52,1.00)^{\star}$ & $1.11(0.80,1.54)$ \\
Coronary heart disease & 26 & $0.56(0.36,0.88)^{\star}$ & $0.52(0.33,0.83)^{\star \star}$ & $1.03(0.65,1.63)$ \\
Non-cardiovascular, smoking related & 28 & $1.08(0.71,1.66)$ & $1.15(0.73,1.79)$ & $0.91(0.58,1.42)$ \\
Cancer & 60 & $0.98(0.74,1.32)$ & $1.04(0.77,1.41)$ & $0.94(0.70,1.26)$ \\
Non-smoking related cancer & 39 & $0.99(0.69,1.42)$ & $1.07(0.74,1.54)$ & $0.95(0.66,1.35)$ \\
Other causes & 38 & $1.00(0.69,1.44)$ & $1.25(0.85,1.83)$ & $0.73(0.50,1.07)$ \\
\hline
\end{tabular}

${ }^{\star} \mathrm{p}<0.05 ;{ }^{\star \star} \mathrm{p}<0.01$.

STATISTICAL METHODS

Age standardised death rates were calculated using person years at risk. All rates were standardised for age at entry to the study by the direct method, using the study population as the standard. The data fulfilled the assumptions for Cox's proportional hazards modelling and such models were used to examine associations between anthropometry and mortality adjusted for possible confounding variables using the SAS PHREG procedure. ${ }^{31}$ To assess the independent effect of trunk length and leg length on overall mortality all models examining the relation between leg length and mortality include trunk length and vice versa.

Height varies between regions in this country and is also affected by socioeconomic factors, diet, birth order, and sex. ${ }^{3}$ Some of these factors are related independently to mortality risk, and may confound relations between height and mortality. ${ }^{32}$ All models were therefore stratified by survey centre and the effects of the following variables were examined in proportional hazards models in male and female subjects separately (a) birth order (the relative ages of family members examined in the survey were used as a proxy for this), (b) total household calorie consumption as a percentage of that recommended by contemporary age and sex specific standards, ${ }^{33}$ (c) social class of head of household using the Registrar General's 1931 classification, (d) the total number of children in the household, (e) a measure of weighted family food expenditure, weighted according to the age and sex of household members, using weightings modified from a 1933 nutritional report, ${ }^{34}$ (f) a measure of current socioeconomic deprivation - the Townsend score ${ }^{34 a}$ - based on census information for the Family Health Service Authority (FHSA) of residence at the time of the patient's embarkation, death or at the end of the follow up period. Age adjustment was carried out using the survey member's age in years and months at the time of the survey. A second variable was also fitted to account for the fact that the survey was carried out over a two year period, so those aged two when examined in 1937 were two years older than those aged two in 1939.

\section{Separate models were fitted examining:}

(1) The age adjusted relation between the anthropometric $\mathrm{z}$ scores, quintiles of these $\mathrm{z}$ scores, and mortality from: all causes; circulatory disease (ICD 390-459); coronary heart 
Table 5A Age adjusted coronary heart disease mortality hazard ratios for men and women by quintiles of leg length and trunk length (the terms for leg length and trunk length were included in the models examining relations between these variables and mortality)

\begin{tabular}{|c|c|c|c|c|c|c|}
\hline \multirow[b]{2}{*}{ Quintile } & \multicolumn{2}{|l|}{ Overall height } & \multicolumn{2}{|l|}{ Leg length } & \multicolumn{2}{|l|}{ Trunk length } \\
\hline & No of deaths & $\begin{array}{l}\text { Hazard ratio } \\
(95 \% \text { CI) }\end{array}$ & No of deaths & $\begin{array}{l}\text { Hazard ratio } \\
(95 \% \text { CI })\end{array}$ & No of deaths & $\begin{array}{l}\text { Hazard ratio } \\
(95 \% \text { CI })\end{array}$ \\
\hline \multicolumn{7}{|l|}{$\operatorname{Men}(n=1128)$} \\
\hline 1 (shortest) & 17 & $1.2(0.6,2.4)$ & 18 & $2.5(1.0,6.2)$ & 16 & $0.9(0.4,1.9)$ \\
\hline 2 & 16 & $1.2(0.6,2.4)$ & 16 & $2.3(0.9,5.6)$ & 13 & $0.7(0.3,1.5)$ \\
\hline 3 & 17 & $1.2(0.6,2.4)$ & 15 & $2.0(0.9,4.9)$ & 18 & $1.0(0.5,2.1)$ \\
\hline 4 & 12 & $0.9(0.4,1.8)$ & 19 & $2.4(1.1,5.4)$ & 15 & $0.8(0.4,1.7)$ \\
\hline 5 (tallest) & 15 & 1.0 & 9 & 1.0 & 15 & 1.0 \\
\hline $\begin{array}{l}\text { Linear trend test } \\
\text { Women }(n=1195)\end{array}$ & & $\mathrm{p}=0.28$ & & $\mathrm{p}=0.14$ & & $\mathrm{p}=0.53$ \\
\hline 1 (shortest) & 12 & $11.2(1.4,87.0)$ & 10 & $3.9(0.8,19.0)$ & 5 & $1.1(0.2,5.3)$ \\
\hline 2 & 4 & $3.7(0.4,33.4)$ & 8 & $3.4(0.7,16.7)$ & 5 & $1.0(0.2,4.6)$ \\
\hline 3 & 4 & $4.1(0.5,36.7)$ & 4 & $1.9(0.3,10.5)$ & 9 & $2.6(0.7,9.8)$ \\
\hline 4 & 5 & $5.1(0.6,44.0)$ & 2 & $0.9(0.1,6.6)$ & 4 & $1.4(0.3,6.5)$ \\
\hline 5 (tallest) & 1 & 1.0 & 2 & 1.0 & 3 & 1.0 \\
\hline Linear trend test & & $\mathrm{p}=0.007$ & & $\mathrm{p}=0.006$ & & $\mathrm{p}=0.97$ \\
\hline
\end{tabular}

Table 5B Age adjusted cancer mortality hazard ratios for men and women (95\% CI) by quintiles of leg length and trunk length (the terms for leg length and trunk length were included in the models examining relations between these variables and mortality)

\begin{tabular}{|c|c|c|c|c|c|c|}
\hline \multirow[b]{2}{*}{ Quintile } & \multicolumn{2}{|l|}{ Overall height } & \multicolumn{2}{|l|}{ Leg length } & \multicolumn{2}{|l|}{ Trunk length } \\
\hline & No of deaths & $\begin{array}{l}\text { Hazard ratio } \\
(95 \% \mathrm{CI})\end{array}$ & No of deaths & $\begin{array}{l}\text { Hazard ratio } \\
(95 \% C I)\end{array}$ & No of deaths & $\begin{array}{l}\text { Hazard ratio } \\
(95 \% \text { CI) }\end{array}$ \\
\hline \multicolumn{7}{|l|}{$\operatorname{Men}(n=1128)$} \\
\hline 1 (shortest) & 7 & $0.4(0.1,0.8)$ & 9 & $0.5(0.2,1.5)$ & 6 & $0.5(0.2,1.4)$ \\
\hline 2 & 13 & $0.7(0.3,1.6)$ & 7 & $0.5(0.2,1.3)$ & 14 & $1.0(0.4,2.2)$ \\
\hline 3 & 7 & $0.5(0.2,1.1)$ & 9 & $0.8(0.3,1.9)$ & 7 & $0.5(0.2,1.4)$ \\
\hline 4 & 8 & $0.5(0.2,1.2)$ & 12 & $0.9(0.4,1.9)$ & 10 & $0.7(0.3,1.7)$ \\
\hline 5 (tallest) & 16 & 1.0 & 14 & 1.0 & 14 & 1.0 \\
\hline Linear trend test & & $\mathrm{p}=0.048$ & & $\mathrm{p}=0.22$ & & $\mathrm{p}=0.51$ \\
\hline \multicolumn{7}{|l|}{ Women $(n=1195)$} \\
\hline 1 (shortest) & 15 & $1.4(0.6,3.1)$ & 12 & $0.9(0.4,2.1)$ & 15 & $1.9(0.8,4.9)$ \\
\hline 2 & 11 & $1.0(0.4,2.3)$ & 14 & $1.0(0.4,2.2)$ & 10 & $1.2(0.4,3.1)$ \\
\hline 3 & 12 & $1.1(0.5,2.7)$ & 12 & $0.9(0.4,2.1)$ & 14 & $1.8(0.7,4.4)$ \\
\hline 4 & 12 & $1.2(0.5,2.8)$ & 10 & $0.8(0.3,1.9)$ & 13 & $1.6(0.7,4.0)$ \\
\hline 5 (tallest) & 10 & 1.0 & 12 & 1.0 & 8 & 1.0 \\
\hline Linear trend test & & $\mathrm{p}=0.94$ & & $\mathrm{p}=0.66$ & & $\mathrm{p}=0.72$ \\
\hline
\end{tabular}

disease (ICD 410-414); non-cardiovascular smoking related illness (cancers of lip - ICD 140 , tongue - ICD 141, mouth and pharynx ICD 143-9, oesophagus - ICD 150, pancreas ICD 157, respiratory tract - ICD 160-3, urinary tract - ICD 188-9, and pneumonia ICD 480-486, bronchitis and emphysema ICD 491,492 and 496, peptic ulcer - ICD 531-534); all malignant neoplasms (ICD 140209); non-smoking related cancers; and other causes of death.

(2) The relation between the above anthropometric variables and all cause and disease specific mortality adjusted for age and the socioeconomic and other variables (a)-(f) listed above.

To examine trends, some tables present hazard ratios by quintile of stature, though unless otherwise stated, all the tests reported are based on the continuous variable for the $\mathrm{z}$ score for the anthropometric index.

Possible clustering effects may arise because several cohort members belong to the same families and therefore share childhood conditions and possible genetic influences on mortality. Therefore separate analyses have been performed using random sampling of one child only per family. This created data sets with 701 boys and 749 girls. Regressions have been performed on 50 such random samples, and the average regression coefficient, weighted by the inverse of the variance of the coefficients from the samples, has been calculated and compared with the results obtained using the whole sample. Fifty six per cent of boys and girls were included in every random sample; this does not pose a problem in relation to the pooled estimate of the regression coefficient itself but it means the standard error of this coefficient cannot be estimated.

\section{Results}

REPRESENTATIVENESS OF TRACED COHORT

MEMBERS

A total of 2547 (85\%) of the children who were examined between the age of 2 years and 14 years 9 months have been traced. Of these, 2519 (98.9\%), were alive and living in Britain on 1 January 1948. Complete information on childhood social circumstances and adult socioeconomic status was available for 1129 of the boys and 1195 girls and they contributed 104113 person years of risk over the 47 year follow up period. Over this time 355 study members died and 65 emigrated. One hundred and three $(29 \%)$ of the deaths were from CHD (77 male and 26 female) and 111 (31\%) were from cancer (51 male and 60 female). Cause of death was not known for one subject.

Men were more likely to have been traced than women, though the difference was small ( $87 \%$ men versus $83 \%$ women $p<0.001)$ and traced survey members were on average one year younger than those who were not traced 
Table 6 Mortality hazard ratios (95\% CI) for coronary heart disease, cardiovascular disease, and cancer for men and women combined (stratified by sex) in relation to a difference of one standard deviation in overall height, leg length, and trunk length examined when aged younger than 8 years or over 8 years at the time of the survey

\begin{tabular}{lllll}
\hline Cause of death & Number of deaths & Height & Leg length & Trunk length \\
\hline Aged $<8$ years $(n=1174)$ & 36 & $0.67(0.47,0.97)^{\star}$ & $0.61(0.41,0.91)^{\star}$ & $1.00(0.70,1.43)$ \\
Coronary heart disease & 57 & $0.81(0.60,1.08)$ & $0.74(0.55,1.00)^{\star}$ & $1.07(0.80,1.44)$ \\
Cardiovascular & 36 & $1.44(1.00,2.08)$ & $1.59(1.06,2.38)^{\star}$ & $1.03(0.70,1.52)$ \\
Cancer & & & & \\
Aged 8+ years $(n=1150)$ & 67 & $0.85(0.66,1.10)$ & $0.81(0.60,1.09)$ & $1.07(0.79,1.43)$ \\
Coronary heart disease & 99 & $0.85(0.69,1.05)$ & $0.81(0.63,1.03)$ & $1.06(0.83,1.35)$ \\
Cardiovascular & 75 & $1.04(0.82,1.32)$ & $1.00(0.76,1.30)$ & $1.07(0.81,1.41)$ \\
Cancer & & & &
\end{tabular}

${ }^{\star} \mathrm{p}<0.05$.

Table 7 Comparison of regression coefficients derived from models including all study members and those derived from pooling the coefficients derived from regression analyses conducted on 50 separate samples each with one randomly selected member of each family (men and women)

\begin{tabular}{llll}
\hline Measure and cause of death & $\begin{array}{l}\text { Pooled regression coefficient on } \\
\text { samples with only one family } \\
\text { member }\end{array}$ & $\begin{array}{l}\text { Regression coefficient } \\
\text { (SE) for whole sample }\end{array}$ & $\begin{array}{l}\text { Difference between coefficients divided } \\
\text { by } \text { of the whole sample regression } \\
\text { coeffient }\end{array}$ \\
\hline $\begin{array}{l}\text { Men } \\
\begin{array}{l}\text { Leg length z score } \\
\text { All cause mortality }\end{array}\end{array}$ & 0.024 & $-0.030(0.086)$ & \\
$\begin{array}{l}\text { Coronary heart disease } \\
\text { All cancers }\end{array}$ & -0.266 & $-0.236(0.141)$ & $0.628 \mathrm{SE}$ \\
$\begin{array}{l}\text { Women } \\
\text { Leg length z score }\end{array}$ & 0.334 & $0.340(0.177)$ & $0.034 \mathrm{SE}$ \\
$\begin{array}{l}\text { All cause mortality } \\
\text { Coronary heart disease }\end{array}$ & 0.042 & & \\
All cancers & -0.621 & $-0.037(0.097)$ & $0.814 \mathrm{SE}$ \\
\hline
\end{tabular}

$(\mathrm{p}<0.001)$. There were no differences in the height, trunk length or leg length $\mathrm{z}$ scores of traced and untraced survey members. Similarly, traced and untraced survey members did not differ with respect to their socioeconomic circumstances or household food consumption.

RELATION BETWEEN ANTHROPOMETRY, CHILDHOOD DIETARY AND SOCIOECONOMIC VARIABLES, AND ADULT SOCIOECONOMIC STATUS Table 1 shows the correlation between dietary and socioeconomic variables and $\mathrm{z}$ scores for overall height, leg length, and trunk length for all survey members, traced and untraced. The correlations between trunk length and most of the socioeconomic variables are low compared with those for leg length and overall height. The correlations with childhood measures of socioeconomic status are higher than those for adult socioeconomic status for which Townsend score is used as a crude proxy.

OVERALL AND CAUSE SPECIFIC MORTALITY IN RELATION TO CHILDHOOD HEIGHT, LEG LENGTH, AND TRUNK LENGTH

Table 2 presents age adjusted death rates per 1000 person years for men and women separately for the main causes of death in relation to quintiles of overall height, leg length, and trunk length. In both men and women rates of cardiovascular disease decrease with increasing height and leg length. In men, cancer mortality tends to increase with leg length.

Table 3 shows the age adjusted hazard ratios for all cause and cause specific mortality in relation to overall height, leg length, and trunk length. This and all subsequent analyses are stratified by survey district. The mortality hazard ratio for each cause of death represents the increase (or decrease) in mortality associated with an increase in one standard deviation score in stature. Models for leg length were adjusted for trunk length and vice versa.

Table 4 presents the same hazard ratios as table 3 but adjusted for indices of childhood socioeconomic deprivation (weighted food expenditure, social class of head of household), birth order, and family calorie consumption as well as adult socioeconomic circumstances (Townsend score). The inverse relation between both height and leg length and CHD mortality in women is little changed. In men the inverse relation between leg length and cardiovascular mortality becomes statistically significant $(\mathrm{p}<0.05)$. In addition men show a significant positive relation between stature and smoking related disease after adjustment for socioeconomic circumstances. The significant association with cancer is little changed. Most of these relations result from differences in leg length.

The relative hazards of CHD mortality and cancer across quintiles of height, leg length, and trunk length are shown in table 5A and 5B. Although there is an decrease in CHD mortality with increasing leg length $(p=0.14$ in men and $p<0.01$ in women), no significant linear trend was found for trunk length. There is a significant relation between overall height and cancer mortality in men $(p=0.048)$ but not women $(p=0.94)$. After adjustment for childhood and adult socioeconomic factors the relation between leg length and cancer in men approaches conventional levels of statistical significance $(\mathrm{p}=0.07)$.

The analyses were repeated separately for those aged less than 8 years $(n=1174)$ and 8 years and over at the time of the survey $(n=1150)$ (table 6). This age was chosen so that there were approximately equal numbers 
of survey members in both age groups and to ensure that all children in the younger age group were pre-pubertal. Because there were few deaths among the younger children this analysis combines male and female subjects and is stratified by sex. The significant relations between anthropometry and cancer and CHD mortality are restricted to those aged $<8$ years. There were 22 cancer deaths in women aged $<8$ years and the hazard ratios $(95 \% \mathrm{CI})$ associated with one standard deviation increase in leg length were $1.92(1.13,3.27)$. Sixteen of the cancer deaths in women were because of breast cancer. In the whole sample the hazard ratios for breast cancer mortality in relation to overall height was 1.1 (0.6 to $2.0 ; \mathrm{p}=0.66)$, for leg length 1.3 (0.7 to $2.2 ; \mathrm{p}=0.42)$, and for trunk length 0.9 ( 0.5 to $1.6 ; \mathrm{p}=0.75)$. In the six cases aged $<8$ years the corresponding figures were for height: $2.1(0.8,5.7 ; \mathrm{p}=0.14)$, leg length, 4.4 $(1.4,13.6 ; \mathrm{p}=0.01)$, and trunk length, 0.6 $(0.2,1.8 ; \mathrm{p}=0.35)$

The analyses for the relation between measures of stature and CHD and cancer mortality were repeated using $\mathrm{z}$ scores derived from external reference populations. ${ }^{29}{ }^{30}$ The main results were generally unaffected although the relation between height and cancer in men was no longer statistically significant (hazard ratio 1.28 (95\%CI 0.97 to 1.69$), \mathrm{p}=0.08$ ).

Fully adjusted proportional hazards analyses were repeated on 50 samples each consisting of one randomly chosen member from each family and the pooled regression coefficients calculated (table 7). The relations between leg length and CHD and cancer mortality did not differ by more than 0.4 standard errors from those in the full models including all survey members, regardless of family membership.

\section{Discussion}

We have found significant relations between leg length in childhood and cardiovascular and cancer mortality in adult men and women. These relations persist after adjustment for childhood and adult socioeconomic circumstances and, in subgroup analyses, are found only in children aged less than eight years. The diminished strength of the relations seen in the older children may be because of differential timing of the adolescent growth spurt as relative height during puberty is related more to the timing of the onset of the adolescent growth spurt than to eventual differences in adult height. The direction of the relations differ: increased leg length was associated with increased cancer mortality but reduced cardiovascular mortality. No significant relations were found with trunk length.

When the regression coefficients were recalculated by pooling the regression coefficients from 50 random samples consisting of only one member from each family these did not differ greatly from those calculated on the full sample. Our results cannot therefore be attributed to shared family patterns of stature and mortality risk. The relation between smoking related illness and anthropometry is in the opposite direction to that for cardiovascular disease indicating that the associations are not as a result of differential adult smoking patterns between height groups. Smoking may, however, confound the height-cancer relation in men.

In 1951, Isabella Leitch when presenting analyses of the Carnegie Survey, reported that leg length was found to be a more sensitive index of socioeconomic circumstances than height. ${ }^{26}$ This has since been supported by other research. ${ }^{17-19}$ Our analyses show that not only does leg length differentiate between socioeconomic groups and environmental conditions, but it also acts as a predictor of future CHD and cancer mortality.

HEIGHT AND CARDIOVASCULAR DISEASE

The risk gradients we found with height in childhood are similar to those reported in adult cohorts. $^{4-9}$ In the US male health professionals cohort, after five years follow up, the relative risk for myocardial infarction was 0.65 in the tallest compared with the shortest men. ${ }^{5}$ In the Nurses Health Study in the US after 14 years of follow up the relative rate of CHD in the tallest $(>1.70 \mathrm{~m})$ compared with the shortest women $(<1.55 \mathrm{~m})$ was 0.73 after adjustment for other risk factors. ${ }^{4}$ Other studies in young adult men followed up over long time periods confirm these findings ${ }^{14}$ although to our knowledge the association between height measured in childhood and CHD mortality has not previously been examined in women. The relations observed in relation to childhood height in the Boyd Orr cohort suggest that the distorting effects of pre-existing disease only partly explains the associations between height and mortality in adults. ${ }^{6}$

The lack of a protective effect for height in relation to non-cardiovascular smoking related mortality suggests that it is unlikely that the differences we have observed are confounded by differences in adult smoking patterns between height groups. We were unable to assess the effects of other possible confounders, such as blood pressure and blood lipids. In adults, if anything, there is an inverse relation between height and both blood pressure and cholesterol. ${ }^{835}$

There are a number of possible explanations for the relation between height and CHD. Firstly, factors that affect growth in childhood-infectious disease exposure, ${ }^{36}$ psychosocial factors, ${ }^{37}$ and diet $^{38}$ - may also be related to the later development of CHD. ${ }^{39} 40$ Adult height trajectories are set early in childhood and early life conditions seem to affect adult height to a greater extent than conditions in later childhood. Height differences between socioeconomic groups are apparent early in childhood and after the first three to four years of life differences in heights between socioeconomic groups remain relatively constant. $^{3641}$ It is therefore possible that the observed relation between height and cardiovascular disease results from factors acting in the first few years of life. These factors also lead to differential leg growth and may be responsible for secular increases in height. ${ }^{317}$ The height-CHD relation was, however, unaffected by adjustments for childhood socioeconomic 
status in these analyses. This may be because leg length is a better marker for the diversity of exposures which affect childhood growth than the measures of childhood socioeconomic status themselves. The measures of calorie consumption, for example, are based on measures of household rather than individual diet. ${ }^{25}$

Secondly, childhood height is related to birthweight and this has been found to be associated with adult CHD. ${ }^{42}$ Two of the studies that have found adult height mortality relations have been able to adjust for birthweight and such adjustments do not significantly alter height-CHD relations. ${ }^{4}$ Ounsted and Moar have studied the growth of babies who were either small, average or large for gestational age. ${ }^{43}$ They found that there was little disproportionality in total length:trunk ratios between the three groups of babies for this measurement. It is therefore possible that differential growth of the legs acts as a marker of conditions in early childhood and that these effects act at a later stage, and independently, of any effects of differential fetal growth upon later disease.

Thirdly, people exposed to the adverse conditions in childhood that retard growth are also more likely to become adult smokers and live in poor socioeconomic circumstances and these in turn are recognised risk factors for cardiovascular disease. ${ }^{44}$ Fourthly, taller children are more likely to experience upward social mobility ${ }^{45}$ and the better socioeconomic circumstances they experience as adults may decrease their risk of disease. In the analyses presented here adjustment for adult socioeconomic conditions did not affect the observed relations. Fifthly, it is possible that genetic factors associated with stature are also related to adult disease. However, twin studies have shown that genetically identical people of differing height also differ in their mortality risk. ${ }^{46}$ Lastly height may act as a marker for coronary artery lumen diameter ${ }^{5}$ and FEV $1 .{ }^{8}$ Both these factors may be related to CHD mortality and, by affecting childhood growth, childhood conditions may indirectly affect adult CHD risk. We found no relation between trunk length and cardiovascular disease, and whereas a relation between trunk length and coronary artery lumen is plausible it seems less probable that leg length is related to coronary diameter independent of trunk length. Similar arguments apply to the suggestion that heightmortality relations result from differences in lung function. ${ }^{8}$ Analyses of data from the Caerphilly study confirm that the association between trunk length and FEV1 is greater than that between leg length and FEV1 (GDS, personal communication). Thus our findings that leg length is the component of stature most closely related to CHD mortality offers little support for mechanical explanations for the height-CHD relation.

The sensitivity of leg length, rather than trunk length, to childhood socioeconomic and dietary factors is reflected in the generally higher correlations observed between leg length and these factors (see table 1). Socioeconomic factors in later childhood have also
KEY POINTS

- Several adult cohorts demonstrate inverse associations between height, a marker for childhood circumstances, and coronary heart disease and positive associations between height and cancer.

- In the Boyd Orr cohort leg length seems to be the component of childhood height most strongly associated with childhood social circumstances and diet.

- Childhood leg length was also inversely associated with coronary heart disease mortality and positively associated with cancer mortality.

- Leg length may be a particularly sensitive marker for childhood diet, infectious disease exposure, and poor living conditions, which may influence the risk of adult disease.

been shown to affect long term health. ${ }^{32} 47$ Although, as in the analyses presented here, adjustments for socioeconomic circumstances in early childhood generally has little effect on adult height-mortality relations. ${ }^{444748}$ It is possible that after the period of height sensitivity in the first few years of life socioeconomic insults are reflected to a lesser extent in height differences. Thus cardiovascular risk may be influenced by exposure to adverse conditions throughout childhood. The observed relation with height may reflect exposures in the first few years of life and the additional association with adverse social circumstances may reflect deprivation during the rest of childhood.

\section{HEIGHT AND CANCER}

We found a positive relation between leg length and cancer in men and women whose height was measured when they were aged less than 8 years. In addition, overall cancer mortality in men of all ages increased with increasing leg length and this relation approached conventional levels of statistical significance after adjustment for childhood socioeconomic status. There were too few cancers in men to examine site specific cancers. In women whose leg length was measured before 8 years of age we found a significant increased risk of breast cancer with increasing leg length, although these analyses were based on only six cases. This supports the findings of Albanes et al who, using data from NHANES, found significant positive linear trends for cancer in men and women in relation to adult height, although these were only statistically significant in men. ${ }^{20}$ In their analysis almost all the observed relation was caused by differences in leg length rather than sitting height (trunk length) ${ }^{20}$ Few studies have examined the relation between cancer and height in childhood or young adults and these mainly have found no significant relation. ${ }^{49-51}$ In contrast several studies of adult cohorts report that breast cancer, colorectal, and overall cancer incidence increases with height. ${ }^{61011}$

The explanation for the positive relation between smoking related mortality and height 
in men is unclear. Most of these deaths were from smoking related cancer. All site cancer mortality tends to be highest in lower socioeconomic groups ${ }^{52}$ and in our study adjustment for socioeconomic status increased the relative risk of cancer associated with increased height. It is unlikely that this relation can be explained by social class changes in smoking habits; although in the past smoking was associated with affluence, social class changes in smoking habits were well underway by the time the subjects were in their $30 \mathrm{~s}$ and $40 \mathrm{~s}^{53}$

One hypothesis for the relation between height and cancer is that excess calorie intake in childhood promotes carcinogenesis. ${ }^{54}$ This is supported by animal studies. ${ }^{55}$ Height, and in particular leg length may act as a marker for childhood diet and excess calorie intake is inferred from tall stature. However, adjustment for family calorie intake in our analyses did not change the strength of the observed relations. In addition to reflecting increased calorie intake taller people also have more cells in their body structure. Albanes and Winnick hypothesise that the associated increase in the number of dividing stem cells, each at risk of undergoing malignant change, places taller people at increased risk of neoplasia. ${ }^{56}$

SOCIOECONOMIC DEPRIVATION IN ADULTHOOD AND OTHER DISEASE RISK FACTORS

We were unable to control for the confounding effects of smoking and other adult lifestyle factors. While it is planned to collect such information in the future we have been restricted to using area of residence as a crude ecological marker for socioeconomic deprivation. It has been shown that the accuracy of socioeconomic classification based on area of residence decreases as the area of analysis increases in size. ${ }^{57}$ The average population size of FHSAs and Health Boards in Britain is around 500000 , thus our adjustments for adult socioeconomic status are imprecise.

\section{Conclusion}

For children who took part in the Carnegie (Boyd Orr) Survey there is an inverse relation between height in childhood and later cardiovascular mortality. This is mainly seen in younger children and is expressed in differences in leg length rather than differences in trunk length. There is a direct relation between childhood stature and cancer-again mainly related to leg length differences. Other research has shown that most of the secular increase in height that occurs in populations is attributable to increases in leg length. It is possible that in these relations leg length is acting as a particularly sensitive marker for the array of dietary, social, and environmental influences that may affect growth in childhood. Our findings suggest that the improved nutrition and living conditions in childhood that are also associated with increases in stature are related to reduced risk of cardiovascular disease and, perhaps, increased risk of cancer in adulthood. It is probable that these influences operate in early childhood.
The authors wish to thank Professor Philip James, director of The Rowett Research Institute for the use of the archive and in particular Walter Duncan, honorary archivist to the Rowett. The staff at the NHS Central Register at Southport and Edinburgh. Sue Williams, Andrea Wilson, Jenny Eachus and Sarah Pike for data entry. Sara Brookes, Phil Chan and Martin Kemp for technical assistance. Dr David Smith for helping us to conact surviving members of the survey teams and for access to his interviews with some of these people. We also wish to acknowledge all the research workers and subjects who participated in the original survey in 19379. In particular we thank Mrs I Crichton and the late David Lubbock for information concerning the design and conduct of the original survey.

Funding: this work was funded by grants from the British Heart Funding: this work was funded by grants from

Connflicts of interest: none.

1 Tanner JM. A history of the study of human growth. Cambridge: Cambridge University Press, 1981

2 Kuh D, Davey Smith G. When is mortality risk determined? Historical insights into a current debate. Social History of Medicine 1993;6:101-23.

3 Rona RJ, Chinn S. Genetic and environmental influences on growth. F Med Screen 1995;2:133-9.

4 Rich-Edwards JW, Manson JE, Stampfer MJ, et al. Height and the risk of cardiovascular disease in women. Am $\mathcal{F}$ Epidemiol 1995;142:909-17.

5 Hebert PR, Rich-Edwards JW, Manson JE, et al. Height and incidence of cardiovascular disease in male physicians. Cirincidence of cardiovascular disease

6 Leon D, Davey Smith G, Shipley M, Strachan D. Adult height and mortality in London: early life, socioeconomic confounding or shrinkage? $\mathcal{F}$ Epidemiol Community Health 1995;49:5-9.

7 Yarnell JGW, Limb ES, Layzell JM, Baker IA. Height: a risk marker for ischaemic heart disease. Eur Heart f 1992;13: 1602-5

8 Walker M, Shaper AG, Phillips AN, Cook DG. Short stature, lung function and risk of a heart attack. Int f Epidemiol 1989; 18:602-6.

9 Watt GCM, Hart CL, Hole DJ, Davey Smith G, Gillis CR, Hawthorne VM. Risk factors for cardiorespiratory and allcause mortality in men and women in urban Scotland: 15 year follow up. Scott Med f 1995;40:108-12.

10 Vatten LJ, Kvinnsland S. Body height and risk of breast cancer. A prospective study of 23,831 Norwegian women. $\mathrm{Br} \mathcal{F}$ cer. A prospective study

11 Waaler HT. Height, weight and mortality: the Norwegian experience. Acta Med Scand 1984;679(suppl): 1-56.

12 Yao CH, Slattery ML, Jacobs DR, Folsom AR, Nelson ET. Anthropometric predictors of coronary heart disease and fatal mortality: findings from the US railroad study. $A m \mathcal{F}$ Epidemiol 1991;134:1278-89.

13 Nieto FJ, Szklo M, Comstock GW. Childhood weight and growth rate as predictors of adult mortality. Am $\mathcal{F}$ Epidemiol 1992;136:201-13.

14 Paffenbarger RSJ, Wing AL. Chronic disease in former college students. X. The effects of single and multiple characteristics on risk of fatal coronary heart disease. Am f Epidemiol 1969;90:527-35.

15 Allebeck P, Bergh C. Height, body mass index and mortality: do social factors explain the association. Public Hortality: do social factors

16 Floud R, Wachter K, Gregory A. Height, health and history. Nutritional status in the United Kingdom, 1750-1980. Nutritional status in the United Kingdom,

17 Tanner JM, Hayashi T, Preece MA, Cameron N. Increase in length of leg relative to trunk in Japanese children and adults from 1957 to 1977: comparison with British and with Japanese Americans. Ann Hum Biol 1982;9:411-23.

8 Billewicz WZ, Thomson AM, Fellowes HM. A longitudinal study of growth in Newcastle upon Tyne adolescents. Ann Hum Biol 1983;10:125-33.

19 Greulich WW. A comparison of the physical growth and development of American- born and native Japanese children. Am $\mathcal{F}$ Phys Anthropol 1957;15:489-515.

20 Albanes D, Jones DY, Schatzkin A, Micozzi MS, Taylor PR. Adult stature and risk of cancer. Cancer Res 1988;48:165862.

21 Chyou H, Nomura AMY, Stemmermann GN. A prospective study of weight, body mass index and other anthropometric measurements in relation to site-specific cancers. Int 7 ric measurements in relatic $1994 ; 57: 313-17$.

22 Damon A, Damon ST, Harpending HC, Kannel WB. Predicting coronary heart disease from body measurements of Framingham males. F Chronic Dis 1969;21:781802 .

23 Higgins ITT, Cochrane AL, Thomas AJ. Epidemiological studies of coronary disease. $\mathrm{Br} \mathcal{F}$ Prev Soc Med 1963;17: $153-65$.

24 Rowett Research Institute. Family diet and health in pre-war Britain. Dunfermline: Carnegie United Kingdom Trust, 1955

25 Gunnell DJ, Frankel S, Nanchahal K, Braddon FEM, Davey Smith G. Lifecourse exposure and later disease: a follow-up study based on a survey of family diet and health in pre-war Britain (1937-9). Public Health 1996;110:85-94.

26 Leitch I. Growth and health. Br f Nutr 1951;5:142-51.

27 Gunnell DJ, Davey Smith G, Frankel SJ, Kemp M, Peters TJ. Socioeconomic and dietary influences on leg length and trunk length in childhood: a re-analysis of the Carnegie 
survey of diet and health in pre-war Britain (1937-9). Paediatr Perinat Epidemiol (in press).

28 Rona RJ, Swan AV, Altman DG. Social factors and height of primary school children in England and Scotland. $\mathcal{F}$ Epide(

29 Tanner JM, Whitehouse RH, Takaishi M. Standards from birth to maturity for height, weight, height velocity, and weight velocity: British children, 1965. Part I. Arch Dis Child 1966;41:454-71.

30 Snyder RG, Schneider LW, Owings CL, Reynolds HM, Golomb DH, Schork MA. Anthropometry of infants, children, and youths to age 18 for product safety design SP-450. New York: Society of Automotive Engineers, 1977.

31 SAS Institute Inc. SAS Technical Report P-217, SAS/STAT Software: The PHREG Procedure. Version 6 ed. Cary, NC, USA: SAS Institute, 1991.

32 Gilksman MD, Kawachi I, Hunter D, et al. Childhood socioeconomic status and risk of cardiovascular disease in middle aged US women: a prospective study. 7 Epidemiol Community Health 1995; 49:10-15.

33 British Medical Association. Report of the Committee on Nutrition. London: British Medical Association, 1950.

34 British Medical Association. Report of Committee Nutrition. London: British Medical Association, 1933.

34a Phillimore P, Beattie A, Townsend P. Widening inequality in health in northern England, 1981-1991. BMF 1994;308 1125-8.

35 Marmot MG, Shipley MJ, Rose G. Inequalities in death specific explanations of a general pattern? Lancet 1984;i 1003-6.

36 Martorell R, Habicht J-P. Growth in early childhood in developing countries. In: Falkner F, Tanner JM, eds. Human growth. A comprehensive treatise.2nd ed. v. 3. New York: Plenum Press, 1986:241-62.

37 Peck MN, Lundberg O. Short stature as an effect of economic and social conditions in childhood. Soc Sci Med 1995;41:733-8.

38 Thomson AM. Fourth Boyd Orr memorial lecture. Problems and politics in nutritional surveillance. Proc Nutr Problems and politics

39 Patel P, Mendall MA, Carrington D, et al. Association of Helicobacter pylori and Chlamydia pneumoniae infections with coronary heart disease and cardiovascular risk factors. BMF 1995;311:711-14

40 Schwartz JE, Friedman HS, Tucker JS, Tomlinson-Keasey C, Wingard DL, Criqui MH. Sociodemographic and psychosocial factors in childhood as predictors of adult mortality. Am F Public Health 1995;85:1237-45.

41 Miller FJW, Billewicz WZ, Thomson AM. Growth from birth to adult life of 442 Newcastle upon Tyne children. $B$ F Prev Soc Med 1972;26:224-30.
42 Barker DJP. Fetal and infant origins of adult disease. London: BMJ Publishing Group, 1992.

43 Ounsted M, Moar VA. Proportionality changes in the first year of life; the influence of weight for gestational age at birth. Acta Paediatr Scand 1986;75:811-18.

44 Notkola V. Living conditions in childhood and coronary heart disease in adulthood. Commentationes Scientiarum Socialium 1985;29:1-119.

45 Power C, Fogelman K, Fox AJ. Health and social mobility during the early years of life. Quarterly fournal of Social Affairs 1986;2:397-413.

46 Vagero D, Leon D. Ischaemic heart disease and low birth weight: a test of the fetal-origins hypothesis from the Swedish Twin Registry. Lancet 1994;343:260-3.

47 Notkola V, Punsar S, Karvonen MJ, Haapakoski J. Socio-economic conditions in childhood and mortality and morbidity caused by coronary heart disease in adulthood in rural Finland. Soc Sci Med 1985;21:517-23.

48 Nystrom Peck AM, Vagero D. Adult body height, selfperceived health and mortality in the Swedish population. 7 Epidemiol Community Health 1989;43:380-4.

49 Le Marchand L, Kolonel LN, Earle ME, Mi M-P. Body size at different periods of life and breast cancer risk. Am $\mathcal{F}$ Epidemiol 1988;128:137-52.

50 Le Marchand L, Wilkens LR, Mi M-P. Early-age body size, adult weight gain and endometrial cancer risk. Int $\mathcal{f}$ Cancer 1991;48:807-11.

51 Greenwald P, Damon A, Kirmss V, Polan AK. Physical and demographic features of men before developing cancer of the prostate. $\mathcal{F}$ Natl Cancer Inst 1974;53:341-6.

52 Davey Smith G, Leon D, Shipley MJ, Rose G. Socioeconomic differentials in cancer among men. Int $\mathcal{f}$ Epidemiol 1991;30:339-45.

53 Todd GF. Social class variations in cigarette smoking and in mortality from associated diseases. Occasional Paper 2. London: Tobacco Research Council, 1976.

54 Albanes D. Caloric intake, body weight, and cancer: a review. Nutr Cancer 1987;9:199-217.

55 Tannenbaum A. Relationship of body weight to cancer incidence. Arch Pathol 1940;30:509-17.

56 Albanes D, Winick M. Are cell number and cell proliferation risk factors for cancer? 7 Natl Cancer Inst 1988;80:772-5.

57 Hyndman JCG, Holman CDJ, Hockey RL, Donovan RJ, Corti B, Rivera J. Misclassification of social disadvantage based on geographical areas: comparison of postcode and collector's district analyses. Int f Epidemiol 1995;24:165- 